\title{
Modelling Morphogenesis: From Single Cells to Crawling Slugs
}

\author{
Nicholas J. Savill* and Paulien Hogeweg† $\dagger$ \\ Theoretical Biology and Bioinformatics, University of Utrecht, Padualaan 8, $3584 \mathrm{CH}$ Utrecht, \\ The Netherlands
}

(Received on 11 January 1996, Accepted in revised form on 11 July 1996)

\begin{abstract}
We present a three-dimensional hybrid cellular automata (CA)/partial differential equation (PDE) model that allows for the study of morphogenesis in simple cellular systems. We apply the model to the cellular slime mold Dictyostelium discoideum "from single cells to crawling slug". Using simple local interactions we can achieve the basic morphogenesis with only three processes: production of and chemotaxis to cAMP and cellular adhesion. The interplay of these processes causes the amoebae to spatially self-organize leading to the complex behaviour of stream and mound formation, cell sorting and slug migration all without any change of parameters during the complete morphogenetic process.

(C) 1997 Academic Press Limited
\end{abstract}

\section{Introduction}

One of the most widely studied and relatively simple organisms in which to study morphogenesis is the cellular slime mold Dictyostelium discoideum. In fact D. discoideum provides an experimentally accessible and relatively simple model for studying many general developmental processes including chemotaxis, cell sorting, pattern formation and complex behaviour through self-organization.

When bacterial food becomes scarce in the soil that they inhabit as unicellular amoebae, they aggregate and form migrating multicellular slugs of two cell types; approximately one fifth prestalk and four fifths prespore cells in the anterior and the posterior of the slug respectively. This process culminates in a fruiting body of spore cells sitting atop a slender tapering stalk. The aggregation is mediated by waves of cAMP originating from centres of aggregation and relayed by the amoebae. The amoebae form streams as they aggregate towards the forming mound of cells at the centre (Bonner, 1982).

There have been many models, continuous, discrete and a combination, covering many aspects of $D$.

*E-mail: njs@ behold.biol.run.nl, †ph@marvel.biol.ruu.nl. discoideum morphogenesis, for example, stream formation (Keller \& Segel, 1970; Mackay, 1978; Parnas \& Segel, 1978; Levine \& Reynolds, 1991; Kessler \& Levine, 1993; Vasiev et al., 1994; Höfer et al., 1995; van Oss et al., 1996), cell sorting (Lacalli \& Harrison, 1978; Meinhardt, 1983; Pate \& Othmer, 1986; Sekimura \& Kobuchi, 1986) and slug migration (Odell \& Bonner, 1986; Williams et al., 1986; Umeda, 1989). These models focus on a number of processes in a given phase of development. Until now no one has described all the phases and the transitions between them in one model. In this paper we present a model that is able to describe uninterrupted basic $D$. discoideum morphogenesis from the aggregation phase to the migrating slug without any change of parameters. This is achieved in a cellular automata (CA). However, amoebae are not just represented by a single automaton but by a group of connected automata forming something like a small blob in the CA. Individual automata can become part of the amoeba or can be removed from the amoeba. In this way one gets a simple form of discrete membrane deformation. The small-scale processes of cell-cell adhesion and chemotaxis are represented as simple rules in the CA. These processes cause the amoebae to self organize and to achieve complex behaviour, i.e. 
stream formation, mound formation, cell sorting and slug migration.

\section{The Model}

We have made use of the cellular automata model of Glazier \& Graner (1993). The beauty of this model is that amoebae are represented as many connected automata instead of a point like object. The amoebae can slide past one another and sort themselves by means of moving their membranes by small amounts. This is caused by small fluctuations around the amoebas' minimized free energy. Several extensions and modifications have been made to the original model: three dimensions, coupling to a partial differential equation (PDE) and chemotaxis up a positive spatial cAMP gradient.

Each amoeba has a unique identification number, $\sigma$. This number corresponds to a state of an automaton in the CA. So any automaton with state 7 , say, is part of amoeba number 7. State 0 does not belong to an amoeba but is considered empty i.e. the medium, in this case the substrate or the air. If there are $N$ amoebae then an automaton can be in $N+1$ states. Amoebae also have associated with them a type, $\tau$, for prespore, prestalk and autocycling prestalk amoebae $(\tau \in\{p, k, a\})$.

All automata on an amoeba's membrane have associated with them a number of dimensionless free energy bonds. The energy bonds connect an automaton with its neighbouring 26 automata. However, the only bonds that contribute free energy to an amoeba are ones that connect automata with different states, that is connect amoebae to one another or to the medium. The magnitude of the bonds depend on the type of the amoebae they connect. This is given by $J_{\tau_{1}, \tau_{2}}>0$ where $\tau_{i}$ are the types of the two amoebae. The bond energy between an amoeba and the medium is given by $J_{\tau, M}$. All other bond energies are 0 . The total free energy of an amoeba due to its surface bond energies is given by:

$$
H=\sum \frac{J_{\text {cell,cell }}}{2}+\sum J_{\text {cell,medium }}+\lambda(v-V)^{2},
$$

where $v$ is the volume of the cell (number of automata), $V$ is the target volume of a cell and $\lambda$ is the inelasticity of an amoeba (i.e. the lower the value of $\lambda$, the easier it is to deform the amoeba's membrane). The final term ensures that the volume of a cell remains close to $V$ otherwise an amoeba's minimum free energy would be zero implying zero volume. Note the equation $\gamma_{\tau_{1}, \tau_{2}}=J_{\tau_{1}, M}+J_{\tau_{2}, M}-J_{\tau_{1}, \tau_{2}}$ gives the energy, and hence is proportional to the force, needed to separate two amoebae. In order to model an amoeba deforming its membrane we use the following rule: an automaton is chosen at random and the state of one of its neighbours is copied into it with a probability of occurring given by:

$$
\begin{aligned}
& \Delta H<-0.1, p .=1, \\
& \Delta H \geq-0.1, p .=e^{-(\Delta H+0.1),}
\end{aligned}
$$

where $\Delta H$ is the change in energy if the copying were to occur. These equations model minimization of an amoeba's free energy. Note that we do not strictly have local conservation of mass in small time periods. However, an average cell volume of $\sim 60$ in the simulations implies only a $1.6 \%$ change in the volume for a single automaton copying step. The value of -0.1 in eqn (2) is to stop the amoebae from sorting when they are not in their movement phase (see below). We chose bond energies so that the amoebae adhere but also so that they will sort themselves into three fairly homogeneous groups given the possibility to do so. We have run simulations where instead of copying one automaton into another we swap them. We observe no change in the results.

The cAMP signalling system is very complex and has been modelled in detail by Martiel \& Goldbeter (1987). However, the cAMP dynamics can be thought of as an excitable medium (Tyson et al., 1989). We use one such model, the system of Panfilov \& Winfree (1985) based on the Fitzhugh-Nagumo equations. Their system allows for greater control of the refractory periods and is much faster to compute than the classical system.

$$
\begin{aligned}
& \frac{\partial c}{\partial t}=D \nabla^{2} c-f(c)-r \\
& \frac{\partial r}{\partial t}=\varepsilon(c)(k c-b-r),
\end{aligned}
$$

where $c$ is the concentration of cAMP, $r$ the refractoriness, $D$ the diffusion coefficient, $f(c)$ a piecewise linear function and $\varepsilon(c), k$ and $b$ are parameters. For some positive values of $b$, a stable limit cycle appears in phase space and we use this to model the cAMP dynamics of the autocycling amoebae. The excitable amoebae have $b=0$.

It is possible that all amoebae autocyclically produce cAMP but the period of the oscillations varies from cell to cell. However, there would be entrainment of the slow cycling amoebae to the faster cycling amoebae. The fastest cycling amoebae would become the centres of aggregation. In the model where we only have a few amoebae due to computational constraints we initialize the system with four autocycling prestalk amoebae in 
the centre of the field. The other amoebae are excitable.

Each automaton in the CA is associated with one lattice point in the discretized numerical PDE. The equations are solved on all lattice points that form part of an amoeba. Diffusion of cAMP occurs on all lattice points containing an amoeba and in the substrate. Zero-flux boundary conditions exist around the ground layer and form a single layer of lattice points around the amoeba-air boundary.

Experiments suggest that an amoeba orients itself towards the aggregation centre in the first $\sim 30 \mathrm{~s}$ after the front of the cAMP wave has reached it; it is not known whether this is caused by the spatial or temporal dynamics of the wave. After orientation the amoebae blindly migrate (Wessels et al., 1992). To incorporate such dynamics into our model would detract from its simplicity and is also not useful. We only require that amoebae migrate a few micrometres with the passing of each wave, it is not important how they do this, just that they do. The natural way to incorporate chemotaxis into the model is to use the spatial gradient of the cAMP wavefront. We do this by modifying the change in energy $\Delta H$, in eqn (2), and hence the probability, of the neighbour copying its state into the automaton as a function of the difference in the cAMP concentrations in the two automata:

$$
\Delta H^{\prime}=\Delta H-\mu\left(c_{\text {automaton }}-c_{\text {neighbour }}\right),
$$

where $\Delta H^{\prime}$ is the new change in energy. This equation implies that an amoeba is more likely to move to a space of higher cAMP concentration and less likely to move to a space of lower cAMP concentration. All amoebae obey this rule and $\mu$ is invariant over all amoebae.

D. discoideum appear to go through several phases between successive cAMP waves. These phases can be roughly described as: stationary and ready to be excited before the cAMP wave, chemotactically moving up the wavefront and producing cAMP and, after the wave top has passed, stationary and refractory. This is modelled in the following way. In an amoeba's refractory and unexcited phases eqn (4) is not applied to the copying probability. Because of the -0.1 in eqn (2) the amoeba is almost completely stationary. As the next cAMP approaches a check is made on the cAMP concentration at all sites occupied by the amoeba. When any site concentration passes 0.1 the amoeba enters its movement phase and eqn (4) is applied. The amoeba moves for a fixed period of 50 time steps, which approximates to the wave peak just passing the amoeba. It then enters its stationary, refractory phase again.
Gravity does not appear to play any significant role in the morphogenetic process. For example in experiments the development of the mound is not affected by turning the petri dish upside down. However, the mound does fall to form the slug and we model this very simply by biasing the copying probability downwards at an angle of $45^{\circ}$ when the time comes for the mound to fall (i.e. when the mound becomes unstable and topples over):

$$
\begin{aligned}
\Delta H^{\prime}=\Delta H+\left(z_{\text {automaton }}-\right. & \left.z_{\text {neighbour }}\right) \\
& +\left(y_{\text {automaton }}-y_{\text {neighbour }}\right),
\end{aligned}
$$

where $z$ and $y$ are the Cartesian coordinates of the automata.

\section{A Simulation}

Cell fate is closely correlated to the cell cycle phase at the onset of development (Zimmerman \& Weijer, 1993). However, at least cAMP, differentiation-inducing factor and ammonia are known to be responsible for the final differentiation during development (Wang \& Schaap, 1989). For simplicity we assign random types to the cells (in the ratio 1 prestalk to 4 prespore cells) just before aggregation occurs. However, we have run simulations where we wait until the mound has partially formed before we assign cell types, and we still observe the same cell sorting due to differential adhesion. This clearly shows how powerful this process can be in pattern formation.

Figure 1(a)-(f) shows the developmental time sequence from aggregation to mound to slug. Streams are formed after several waves (Fig. 2). The autocycling amoebae are pushed upwards by incoming amoebae leading to the formation of the growing mound. By differential adhesion some of the prestalk cells are slowly sorted into the anterior end of the mound. After 25 waves of cAMP we apply "gravity" and the mound falls over. The multicellular organism then crawls on the ground by cAMP waves diffusing along its length.

\section{Stream Formation}

Stream formation has been shown to occur by a positive feedback mechanism (Keller \& Segel, 1970; Levine \& Reynolds, 1991; Vasiev et al., 1994): high cell density causes instability in the cAMP wavefront so that the resulting wavefront curvature causes cells to migrate towards the high density region hence increasing the density and the wavefront instability. However, the instability is induced only if the intra-cellular cAMP production is fast enough to cause the wave velocity to be proportional to the cell 

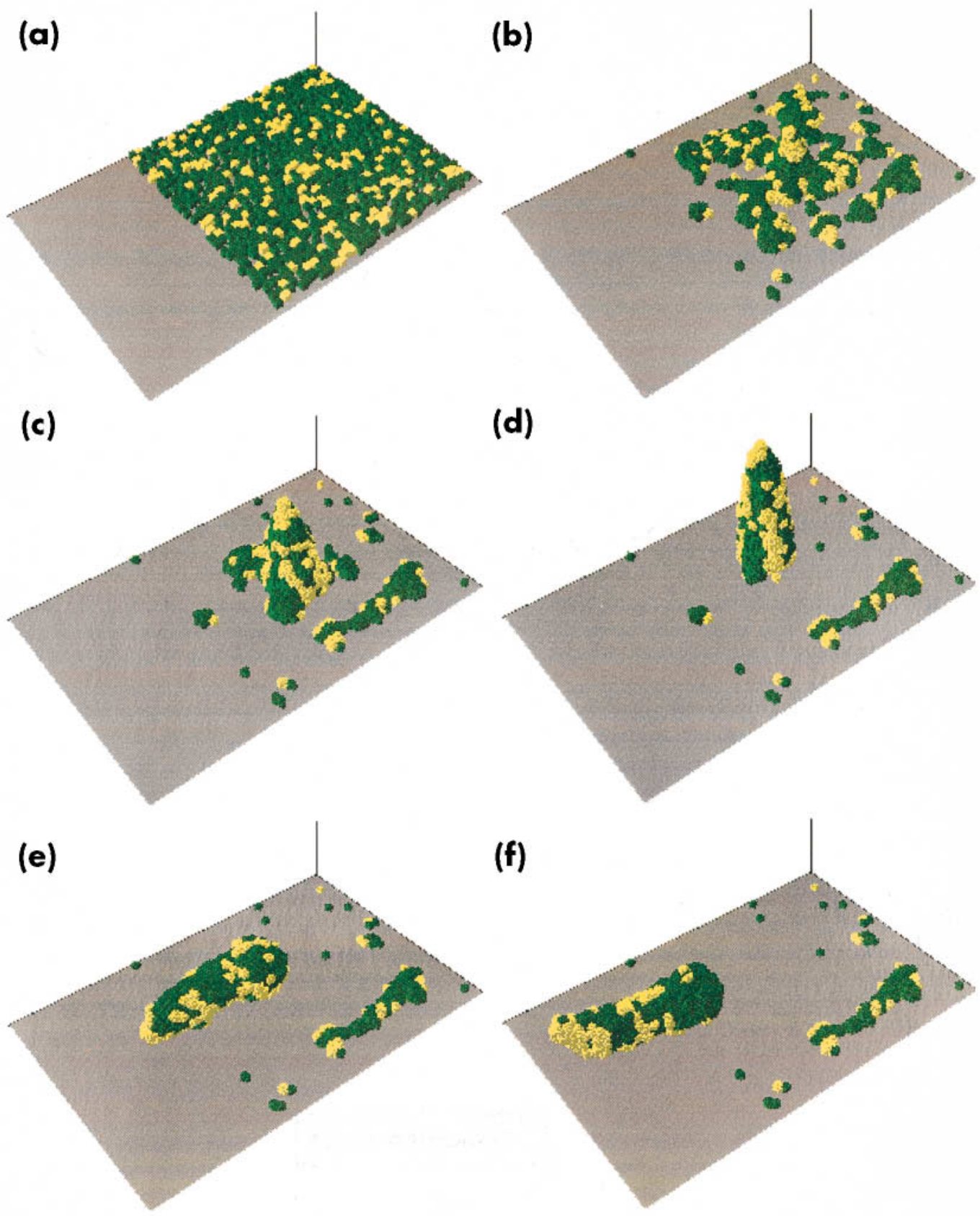

FIG. 1. Time sequence of the aggregation, stream formation, mound, falling and slug phase of a simulation of Dictyostelium. (a) Initial random distribution of 832 cells ( 4 autocycling in the centre, $20 \%$ prestalk (yellow), $80 \%$ prespore (green)) giving $59 \%$ coverage or $9 \times 10$ amoebae $/ \mathrm{cm}^{2}$. Grey is the ground layer. (b) After 50 min (7 cAMP waves) the mound has started to form and streams are established. (c) After 100 min (14 waves) most amoebae are now in the mound. (d) After 150 min (21 waves) the mound has fully formed. (e) After $200 \mathrm{~min}$ (28 waves) the mound has almost fallen to the ground. (f) After $350 \mathrm{~min}$ (50 waves) the slug is migrating. Aggregation is done on a field size of $150 \times 150 \times 100$ and all amoebae are initiated as a $4 \times 4 \times 4$ automata cube, although they quickly round up due to energy minimization. One time step (solution of the PDE) corresponds to about 0.3 seconds and one grid point to $8 \mu \mathrm{m}^{3}$. There are the same number of automata swaps as there are automata in the field for every time step. Cell types are $\tau \in\{a, k, p\}$ where $a$ is autocycling prestalk, $k$ is prestalk and $p$ is prespore. Bond energies are $J_{a, a}=3, J_{k, k}=3, J_{p, p}=4, J_{a, M}=3, J_{k, M}=2, J_{p, M}=2, J_{a, k}=J_{k, p}=J_{a, p}=4$. V $=68$, $\mu=3$. The parameters and function used for the Fitzhugh-Nagumo equations are $f(c)=C_{1} c,-C_{2} c+a, C_{3}(c-1)$ and $\varepsilon(c)=\varepsilon_{1}, \varepsilon_{2}, \varepsilon_{3}$ for $c<c_{1}, c_{1}<c<c_{2}, c_{2}<c$ respectively. $C_{1}=20, C_{2}=3, C_{3}=15, a=0.15, c_{1}=0.0065, c_{2}=0.841, D=1, \varepsilon_{1}=0.5, \varepsilon_{2}=0.0589, \varepsilon_{3}=0.5$, $k=3.5$. The PDE equations are solved by the explicit Euler method (with time step equal to 0.01 and space step equal to 0.37). Diffusion is calculated using the central difference method. 


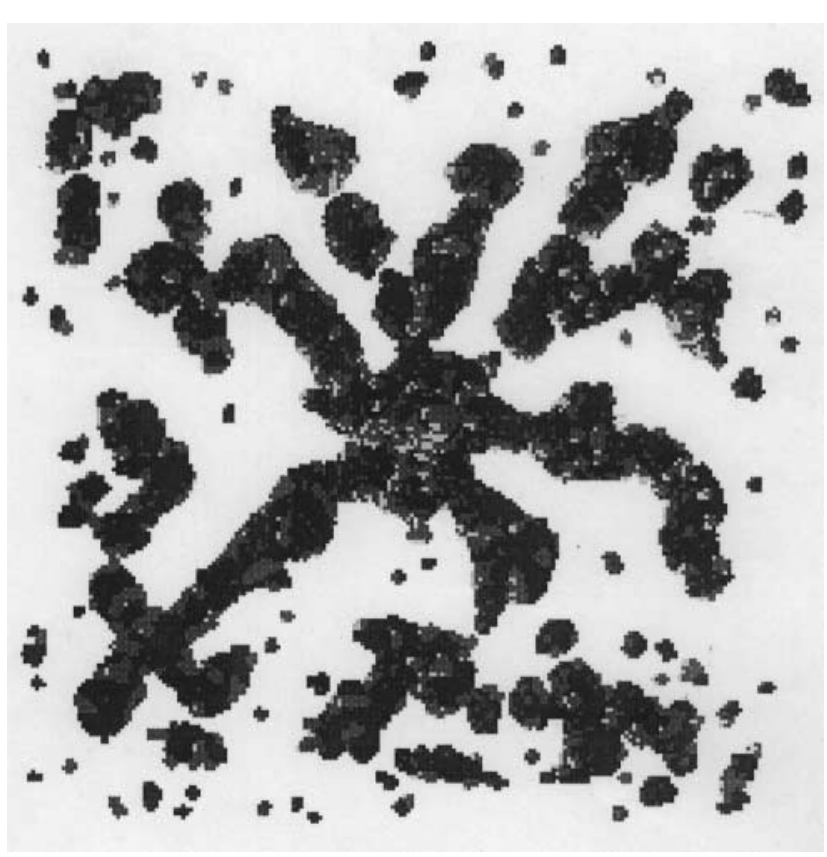

FIG. 2. An overhead view of stream formation on a $300 \times 300$ grid. The parameters are the same as those described in the legend to Fig. 1.

density (van Oss et al., 1996). So why should $D$. discoideum form streams in the first place?

In Fig. 3(a) and (b) we show an experiment in which we measure the velocity of a group of amoebae and a single amoeba. Figure 4 shows the velocity of the group and the individual for varying values of $J_{a, M}$. The figures clearly show that if amoebae adhere they will move faster than if they remain separated. Intuitively this can be understood in the following way. An amoeba in the centre of a group of amoebae, when it moves, not only changes its own membrane

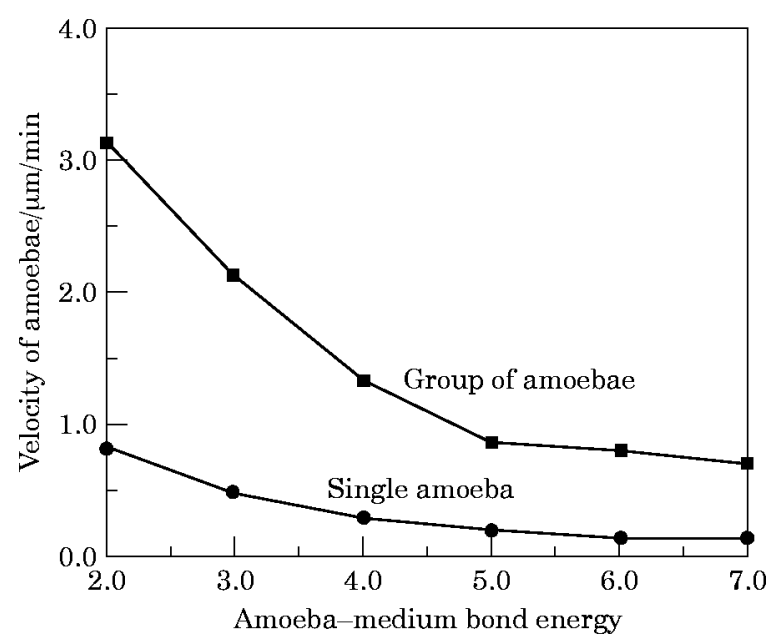

FIG. 4. Plot of velocity (each point averaged over 10 simulations) against the cell-medium bond energy for prespore amoebae. Given that the amoebae adhere to each other the group will always move faster than a single amoeba. Parameters are as described in the legend to Fig. 1. $\mathbf{\square}$, Group of amoebae; $\boldsymbol{0}$, single amoeba.

and volume but also its neighbour's membrane and volume. The resulting deviation from the energy minimum is larger than for membrane deformation of an individual amoeba. The consequent relaxation via chemotaxis to the minimum energy is therefore more probable in the group and hence the group moves with a faster speed. It is known that once streams are formed the frequency of the cAMP waves increases (e.g. Gross et al., 1976), which could also increase the average velocity of amoebae within the streams. But in simulations we find this effect is much weaker than the effect of adhesion. Hence we propose that the reason why streams are formed is so that amoebae can use their
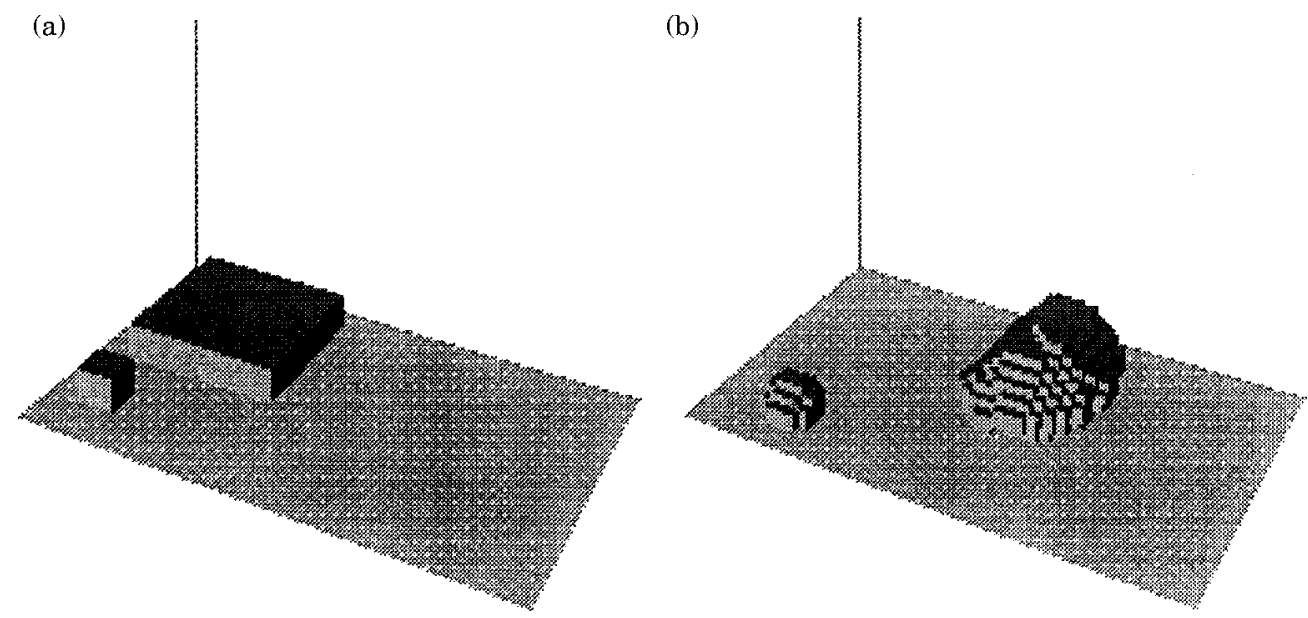

FIG. 3. The velocity of the group of prespore amoebae is greater than that of a single amoeba. (a) A group of 16 amoebae and an individual are placed at the end of a plane. cAMP diffuses in plane wave pulses of fixed period from the other end of the plane. (b) After 7 cAMP waves the group has chemotactically moved a further distance than the single amoeba. All parameters are as described in the legend to Fig. 1. 
adhesiveness to aggregate faster, a selective advantage in harsh conditions.

\section{Cell Sorting}

The relative roles of positional information and cell sorting in $D$. discoideum is still an open question although much progress has been made in isolating morphogens and illucidating their regulation (Kay et al., 1989; Williams et al., 1989). They show that both processes seem to play a role in the patterning of the different types of prestalk cells.

Figure 5 shows that pattern formation is occurring in the model. We plot the percentage of prestalk cells in the anterior end of the mound and slug compared to those prestalk cells that are not in the anterior. The patterns occur via cell sorting due to differential adhesion only. This result confirms the experiments of Sternfeld (1979) and Springer \& Barondes (1978) and the model of Sekimura \& Kobuchi (1986).

We show that cell sorting is generated by the dynamics of our model. In future work we hope to show that positional information may also be generated by the dynamics: cells do not all starve at exactly the same time and hence have maturated for different periods. We postulate that the maturation involves both the cAMP dynamics (in particular the parameter $b$, which shifts the cAMP dynamics from non-responsive to relaying to autocycling) and the increase in cell-cell adhesion. Cells that matured the earliest or are in a specific phase of the cell cycle excrete cAMP first and form the tip of the mound and so "organize" the aggregation. If we assume that the maturation process is slowed down by receiving

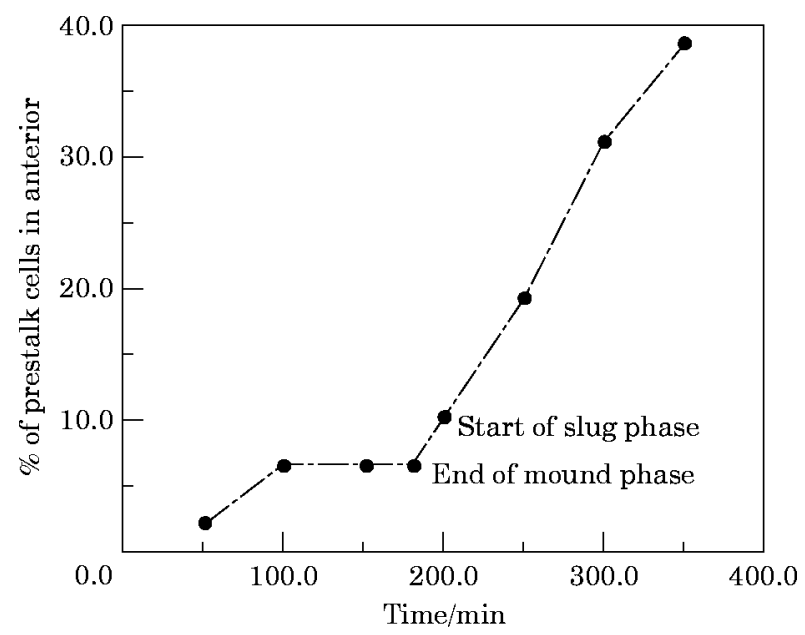

FIG. 5. A rough percentage of the number of prestalk cells that form a solid mass at the anterior of the mound and slug, compared to those prestalk cells that are still present in the prespore region.
cAMP signals or accompanying clues, cells that began maturing a little later may be the prestalk cells first recognizable in the mound and cells that matured the latest may be the prespore cells. Hence cell differentiation would be accomplished without any further assumptions. Moreover we could expect the regeneration of prestalk and prespore zones if the tip of the mound/slug is removed as is observed in experiments.

\section{Slug Migration}

Two slug migration models have been proposed; the fountain flow of Odell \& Bonner (1986) proposes differential motion of the amoebae in the core and the periphery of the slug and the squeeze-pull model of Williams et al. (1986) proposes that circumferential cells squeeze forward a circular core followed by pulling up of the rear. Even though our model does not include the physical forces involved in slug migration our results suggest a rather simpler explanation than those described in the above articles and is also in agreement with the experiments of Siegert \& Weijer (1992).

A pulse of cAMP is initiated in the anterior of the slug. However, because all autocycling amoebae produce cAMP at the same instance they see no positive cAMP gradient. In other words they do not chemotactically move. On the other hand the prestalk amoebae just behind them see the positive cAMP gradient of the pulse. These amoebae move forward and in doing so push the autocycling amoebae forward. The cAMP wave propagates backwards through the slug causing all amoebae to move forward. This causes a ripple effect as amoebae move forward from high to low density areas that were just vacated by the amoebae in front of them. Over many cAMP waves the slug is seen to crawl forward in small steps.

Siegert \& Weijer (1992) observe prespore amoebae moving periodically and always forward. They conclude that "slug movement and morphogenesis can be simply explained by the same principles that govern aggregation i.e. wave propagation and chemotaxis", a conclusion that is verified by our model.

\section{Conclusion}

We demonstrate a simple model that can, with no change of parameters, describe the basic morphogenesis, in three dimensions, of $D$. discoideum "from single cells to crawling slug". Compared to previous models that only consider phase specific processes, we 
show that only three processes are needed to give the basic morphogenesis. These processes are production of and chemotaxis to cAMP and cellular adhesion. The interplay of these three basic behaviours cause the amoebae to organize themselves spatially. In other words form streams, build a mound, sort into two cell types, fall over and crawl away. These higher behaviours, even though complex, are readily studied and explained because of their simple foundations. The reason why this model, in comparison with previous models, can produce these behaviours is because of the interactins on a scale smaller than the diameter of the amoebae. That is to say that the amoebae membranes are deformed to minimize their free energy.

Of course, we cannot explain the finer details of the morphogenesis, and we do not wish to. Our model provides the substrate for morphogenesis on which the cells' higher functions can operate (Boerlijst \& Hogeweg, 1991).

Our model makes several predictions about the behaviour of $D$. discoideum amoebae that can be tested experimentally. Cells in aggregates move faster than when alone. This observation suggests an evolutionary explanation for stream formation, which occurs if and only if there is fast intra-cellular cAMP production. The sorting of cell types can be partially or fully due to differential adhesion between the cell types. Siegert \& Weijer (1992) were able to observe, with high resolution digital image processing, circular motion of individual amoebae in the anterior of slugs that produce scroll waves of cAMP. We predict that for slugs of strains not producing spiral and scroll waves that all amoebae will be seen to move with the same average speed and direction compared to that of the whole slug.

This method of cell modelling is widely applicable to many areas of cell biology as well as more diverse fields (Savill \& Hogeweg, 1996).

We would like to thank Arjan van de Merwe for helpful discussions. N. Savill is supported by the Priority Program Nonlinear Systems of the Netherlands Organization for Scientific Research. The Dynamics Systems Lab. provided the computer facilities.

\section{REFERENCES}

Boerlisst, M. C. \& Hogeweg, P. (1991). Self-structuring and selection: Spiral waves as a substrate for evolution. In: Artificial Life II (Langton, C. G., ed.) pp. 255-276. Redwood City: Addison-Wesley.

BONNER, J. T. (1982). Comparative biology of cellular slime molds. In: The Development of Dictyostelium discoideum (Loomis, W., ed.) pp. 1-33. New York: Academic Press.

Glazier, J. A. \& Graner, F. (1993). Simulation of the differential driven rearrangement of biological cells. Phys. Rev. E 47, $2128-2154$

Gross, J. D., Peacey, M. J. \& Trevan, D. J. (1976). Signal emission and signal propagation during early aggregation in Dictyostelium discoideum. J. Cell Sci. 22, 645-656.

Höfer, T., Sherratt, J. A. \& Maini, P. K. (1995). Dictyostelium discoideum: Cellular self-organization in an excitable biological medium. Proc. Roy. Soc. Lond. Ser. B. 259, 249-257.

Kay, R. R., Berks, M. \& Traynor, D. (1989). Morphogen hunting in Dictyostelium. Development 67 (Suppl.), 81-90.

Keller, E. F. \& Segel, L. A. (1970). Initiation of slime mold aggregation viewed as an instability. J. theor. Biol. 26, 399-415.

Kessler, D. A. \& Levine, H. (1993). Pattern formation in Dictyostelium via the dynamics of cooperative biological entities. Phys. Rev. E 48, 4801-4804.

LaCAlli, T. C. \& Harrison, L. G. (1978). The regulatory capacity of Turing's model for morphogenesis with application to slime molds. J. theor. Biol. 70, 273-295.

Levine, H. \& ReYNolds, W. (1991). Streaming instability of aggregating slime mold amoebae. Phys. Rev. Letters 66, 2400-2403.

MACKAY, S. A. (1978). Computer simulation of aggregation in Dictyostelium discoideum. J. Cell Sci. 33, 1-16.

Martiel, J. \& Goldbeter, A. (1987). A model based on receptor desensitization for cyclic AMP signalling in Dictyostelium cells. Biophys. J. 52, 807-828.

MeinhaRdT, M. (1983). A model for the prestalk/prespore patterning in the slug of the slime mold Dictyostelium discoideum. Differentiation 24, 191-202.

Odell, G. M. \& Bonner, J. T. (1986). How the Dictyostelium discoideum grex crawls. Phil. Trans. Roy. Soc. Lond. Ser. B 312, 487-525.

Panfilov, A. V. \& Winfree, A. T. (1985). Dynamical simulations of rotational scroll rings in 3D excitable media. Physica D 17, 323-330.

Parnas, H. \& Segel, L. A. (1978). A computer simulation of pulsatile aggregation in Dictyostelium discoideum. J. theor. Biol. 71, 185-207.

Pate, E. F. \& Othmer, H. G. (1986). Differentiation, cell sorting and proportion regulation in the slug stage of Dictyostelium discoideum. J. theor. Biol. 118, 118-319.

SAVILL, N. J. \& HogeweG, P. (1996). A cellular automata approach for modeling cell-cell interactions. In: Dynamics of Cell and Tissue Motion (Alt, A., Deutsch, A. \& Dunn, G., eds) in press. Basel: Birkhäuser Verlag.

Sekimura, T. \& Kobuchi, Y. (1986). A spatial pattern formation model for Dictyostelium discoideum. J. theor. Biol. 122, 325-338.

Siegert, F. \& WeiJer, C. J. (1992). Three dimensional scroll waves organise Dictyostelium slugs. Proc. Natl. Acad. Sci. U.S.A. 89, 6433-6437.

SPRINGER, W. R. \& BARONDES, S. H. (1978). Direct measurement of species specific cohesion in cellular slime molds. J. Cell Biol. 79, 937-942.

STERNFELD, J. (1979). Evidence for differential cellular adhesion as the mechanism of sorting out of various slime mold species. $J$. Embryol. Exp. Morphol. 53, 163-177.

Tyson, J., Alexander, K., Manoranjan, V. \& Murray, J. (1989). Spiral waves of cyclic AMP in a model of slime mold aggregation. Physica D 34, 193-207.

UMEDA, T. (1989). A mathematical model for cell sorting, migration and shape in the slug stage of Dictyostelium discoideum. Bull. Math. Biol. 51, 485-500.

van Oss, C., Panfilov, A. V., Hogeweg, P., Siegert, F. \& Weijer, C. J. (1996). Spatial pattern formation during aggregation of the slime mold Dictyostelium discoideum. J. theor. Biol. 181, 203-213.

Vasiev, B. N., Hogeweg, P. \& Panfilov, A. V. (1994). Simulation of Dictyostelium discoideum aggregation via reaction-diffusion model. Phys. Rev. Letters 73, 3173-3176.

Wang, M. \& SchaAp, P. (1989). Ammonia depletion and DIF trigger stalk cell differentiation in intact Dictyostelium discoideum slugs. Development 105, 569-574. 
Wessels, D., Murray, J. \& Soll, R. (1992). Behaviour of Dictyostelium amoebae is regulated primarily by the temporal dynamic of the natural cAMP wave. Cell Motil. Cytoskel. 23, $145-156$.

Williams, J. G., Jermyn, K. A. \& Duffy, K. T. (1989). Formation and anatomy of the prestalk zone of Dictyostelium. Development 107 (Suppl.) 91-97.
Williams, K. L., Vardy, P. H. \& Segel, L. A. (1986). Cell migrations during morphogenesis: Some clues from the slug of Dictyostelium discoideum. Bioessays 5, 148-151.

Zimmerman, W. \& WeiJer, C. J. (1993). Analysis of cell cycle progression during the development of Dictyostelium and its relationship to differentiation. Dev. Biol. 160, 178-185. 